\title{
Psychophysical functions for active tactual discrimination of line width by blind children
}

\author{
EDWARD P. BERLA' and MARVIN J. MURR \\ American Printing House for the Blind, Louisville, Kentucky 40206
}

\begin{abstract}
Two psychophysical experiments were conducted on active tactual discrimination of line width using the method of constant stimuli with blind subjects. In Experiment I, 93 subjects made single judgments over each of five standards between 0.04 and 0.12 in. In Experiment II, 6 subjects were tested repeatedly over seven standards between 0.04 and $0.50 \mathrm{in}$. Over the range of standards common to both experiments, the Weber fractions decreased as the width of the standard increased. For two larger standards ( 0.25 and $.50 \mathrm{in}$.), the Weber fractions approached asymptote and then increased respectively suggesting a $\mathrm{U}$-shaped function. No differences in accuracy of discrimination were found between males vs. females, age groupings, or grade groupings.
\end{abstract}

Visually handicapped students use their fingertips to acquire information from tactile displays, either from braille characters (Fertsch, 1947; Nolan \& Kederis. 1969) or from nonpunctiform codes, such as the symbology used for maps (Berla', 1972). A primary need in the education of these students is for readable tactile displays (maps, graphs, and diagrams). A method frequently used for designing tactile displays is to construct a tactile analogue of a visual display. However, given the disparity in resolving power between fingertips and eye, the results may be a symbology that is not discriminable by touch. For example, it has been reported that students cannot recognize basic geometric forms and patterns when they are reduced below $.50 \mathrm{in.}$ $(1.27 \mathrm{~cm}$ ) in diam (Morris \& Nolan, 1963; Zigler \& Northrup, 1926). There is, however, a dearth of information on the tactual discriminatory capacity of the active fingertip(s) for a variety of tactile parameters.

The present investigation was concerned with the active tactual discrimination of line width by blind subjects. The stimuli were raised lines oriented perpendicularly away from the body. While the subject moved his index fingertip toward and away from the body along the length of the line, he had to judge the width of the line. A method of constant stimuli was used. In the first experiment, the subjects were limited in the amount of time they could

The authors extend their appreciation to Mr. George Whetstone. Superintendent of the W. Ross MacDonald School in Ontario, Canada, and to Mr. Will Evans. Superintendent of the Kentucky School for the Blind. We also extend our appreciation to $\mathrm{Mr}$. John Siems, Assistant Data Processing Manager at the American Printing House for the Blind, for his aid in processing the data in this study, and Mr. Lawrence H. Butterfield, Jr., for drawing Figure 1. Requests for reprints may be sent to Edward P. Berla', American Printing House for the Blind, P.O. Box 6085, Lou isville. Kentucky 40206. participate; therefore, a large number (93) of subjects was used, each receiving each comparison pair once. In the second experiment, a repeated-trials procedure was used in which 6 subjects were repeatedly tested over a period of 8 weeks.

\section{EXPERIMENT I}

\section{Method}

Subjects. The subjects were 93 braille readers enrolled in the Ontario School for the Blind. The means and standard deviations of the ages of the students in each of three grade groupings (4-6, 7-9, 10-12) were, respectively: $M=11.94, S D=1.42 ; M=15.73, S D$ $=2.12 ; \mathrm{M}=18.49, \mathrm{SD}=1.70$. There were approximately twice as many males as females in each grade grouping, with $28-33$ subjects within each grade grouping.

Materials. Raised lines were made from cold rolled steel cut with a die and milled to the specified widths. The lines were polished with fine steel wool to reduce burrs. There were five standard line thick nesses of $.04, .06, .08, .10$, and .12 in $(.10, .15, .20, .25$, and $.30 \mathrm{~cm}$ ). Each standard line was paired with 13 variable line widths. Six of the variable line widths were wider than the standard and six were thinner than the standard. For the $.04-\mathrm{in} .(.10 \mathrm{~cm})$ standard, variable widths were separated by $.004-$ in. $(.01 \mathrm{~cm})$ steps; for the other standards, the separation was $.005 \mathrm{in} .(.013 \mathrm{~cm})$. These values were chosen on the basis of a pilot study. One variable line width was equal in width to each standard. All of the raised lines were $2.5 \mathrm{in} .(6.35 \mathrm{~cm})$ in length and were inserted in wooden blocks $4 \times 3$ $\mathrm{x} 1 / 2$ in. $(10.16 \times 7.62 \times 1.27 \mathrm{~cm})$ with approximately $.25 \mathrm{in}$. $(.64 \mathrm{~cm})$ of the metal raised above the surface. The lines were spaced 2 in. $(5.08 \mathrm{~cm})$ apart on a block.

Design and Procedure. The method of constant stimuli was used. The subject was presented with the standard and a variable line width and asked to indicate the wider of the two by tapping it with his finger. He inspected each line separately, using the index finger of the preferred hand. The lines were oriented perpendicular to the body, and the subject traced a line with a motion toward and away from the body. He had free access to both lines of a comparison pair for up to $1 \mathrm{~min}$, but of necessity could feel only one line at a time. However, no subject required the full 1 min. A within-subjects design was used, with each standard-variable pair given once to each subject. A series of 20 practice trials, consisting of the five standards with each of four different variable stimuli drawn randomly from the regular series of 65 stimulus pairs, preceded the 65 pair-comparison trials. 
On approximately halt the trials, the standard was presented on the left. and on halt the trials. on the right.

\section{Results}

A tabulation of the total number of correct responses was made for each subject collapsed across standards (trials in which the standard was compared with itself were eliminated). These data were used in two analyses of variance using Snedecors unweighted means analysis for unequal $\mathbf{n}$ in order to determine whether differences in accuracy existed between sex groupings, grade groupings, or age groupings. In the first analysis, the two variables were sex (male vs. female) and age groupings (young, intermediate, older). There were no significant differences between sexes $(F=1.23 ; \mathrm{df}=1.87 ; \mathrm{p}>.05)$, age groupings $(\mathrm{F}=2.53 ; \mathrm{df}=2,87 ; \mathrm{p}>.05)$, or the interaction of Sex by Age $(F=.42 ;$ df $=2.87 ; p>.05)$. In the second analysis, the two variables were sex (male vs. fenuale) and grade groupings (4-6, 7-9, 10-12). There were no significant differences between sex $(F=1.58$; $\mathrm{df}=1.87 ; \mathrm{p}>.05)$, grade groupings $(\mathrm{F}=1.76 ; \mathrm{df}=$ $2.87 ; \mathrm{p}>.05)$, or Sex by Grade grouping $(\mathrm{F}=2.13$; $\mathrm{dt}=2.87 ; \mathrm{p}>.05)$.

Since there were no significant differences between grades, ages, or sex, the data were combined for each standard and psychometric functions were obtained by the method of least squares. Figure 1 shows the psychometric functions for each standard, with the abscissa showing the width of each line in thousandths of an inch and the ordinate showing the obtained percentage of subjects (93). converted to $\mathrm{z}$ scores, judging the variable line as being wider than the standard. Inspection of the functions shows that the PSEs for each standard were relatively unbiased and the resolving power of the index finger quite good over the range of standards used. The correlations between the obtained percentages converted to $\mathrm{z}$ scores and the line of best tit ranged from $r=.96$ to $r=.99$. showing excellent fit to a straight-line function.

The $50 \%$ and $90 \%{ }^{1}$ difference thresholds were obtained by use of the regression equations (Kling \& Riggs. 1971. p. 29). Weber fractions were also computed for the $50 \%$ and $90 \%$ thresholds and are shown in Table 1 . Inspection of Table 1 shows that. for both the $50 \%$ and $90 \%$ thresholds, the proportion of change necessary to discriminate a line width as greater than the standard decreased as the standard became larger. For the smallest standard [.04 in. $(.10 \mathrm{~cm})]$. the variable needed to be approximately $20 \%$ greater than the standard at the $50 \%$ threshold and $48 \%$ greater than the standard at the $90 \%$ threshold. For the largest standard $[.12 \mathrm{in} .(.30 \mathrm{~cm})]$, the variable needed to be approximately $11 \%$ greater than the standard at the $50 \%$ threshold and $27 \%$ greater than the standard at the $90 \%$ threshold.

\section{Discussion}

Considering the unusual procedure (i.e., the percentage of subjects judging the variable rather than the more typical percentage of responses) used to generate the psychophysical functions, the data were remarkably consistent. The marked consistency obtained with this procedure could be attributed to the fact that blind children have a history of making "fine" tactual discriminations and that this transferred to the line width discrimination task. Consequently, the results suggest that the somewhat unusual procedure may be a feasible method for obtaining psychophysical data on other tactile parameters with subject populations where there are limitations on testing time.

For the conventional $50 \%$ threshold as well as the $90 \%$ threshold, there were consistent decreases in the Weber fractions as line width increased. The largest Weber fraction (the smallest standard) was 1.82 times greater than the smallest Weber fraction (largest standard) for both the $50 \%$ and $90 \%$ thresholds. Weber's law did not hold within the range of stimuli used here, and there was notable consistency in the departure from the law; namely, the wider the line, the smaller the relative increase in a variable line that can be discriminated as wider.

\section{EXPERIMENT II}

The purpose of the second experiment was to extend Experiment I to include the larger line widths of $.25 \mathrm{in} .(.64 \mathrm{~cm})$ and $.50 \mathrm{in} .(1.27 \mathrm{~cm})$. and to use a repeated-trials procedure with a few subjects in order to compare the results with the many-subjects procedure used in Experiment $\mathbf{I}$.

\section{Method}

Subjects. The subjects were three male and three female braille readers enrolled in the Kentucky School for the Blind. Each subject's age, sex. grade level. and visual acuity, as obtained from ophthalmological reports. is shown in Table 2.

Materials. The same tive sets of raised lines were used as in the

Table 1

Weber Fractions for the $50 \%$ and $90 \%$ Thresholds for Each Standard for Both Experiments I and II

\begin{tabular}{ccccc}
\hline & \multicolumn{2}{c}{$50 \%$} & \multicolumn{2}{c}{$90 \%$} \\
Standard & I Experiment & II & I Experiment \\
\hline .040 & .1987 & .1560 & .4834 & .3797 \\
.060 & .1647 & .1275 & .4007 & .3101 \\
.080 & .1305 & .1028 & .3175 & .2502 \\
.100 & .1117 & .0907 & .2717 & .2207 \\
.120 & .1093 & .0850 & .2660 & .2069 \\
.250 & & .0830 & & .2020 \\
.500 & & .0967 & & .2353 \\
\hline
\end{tabular}



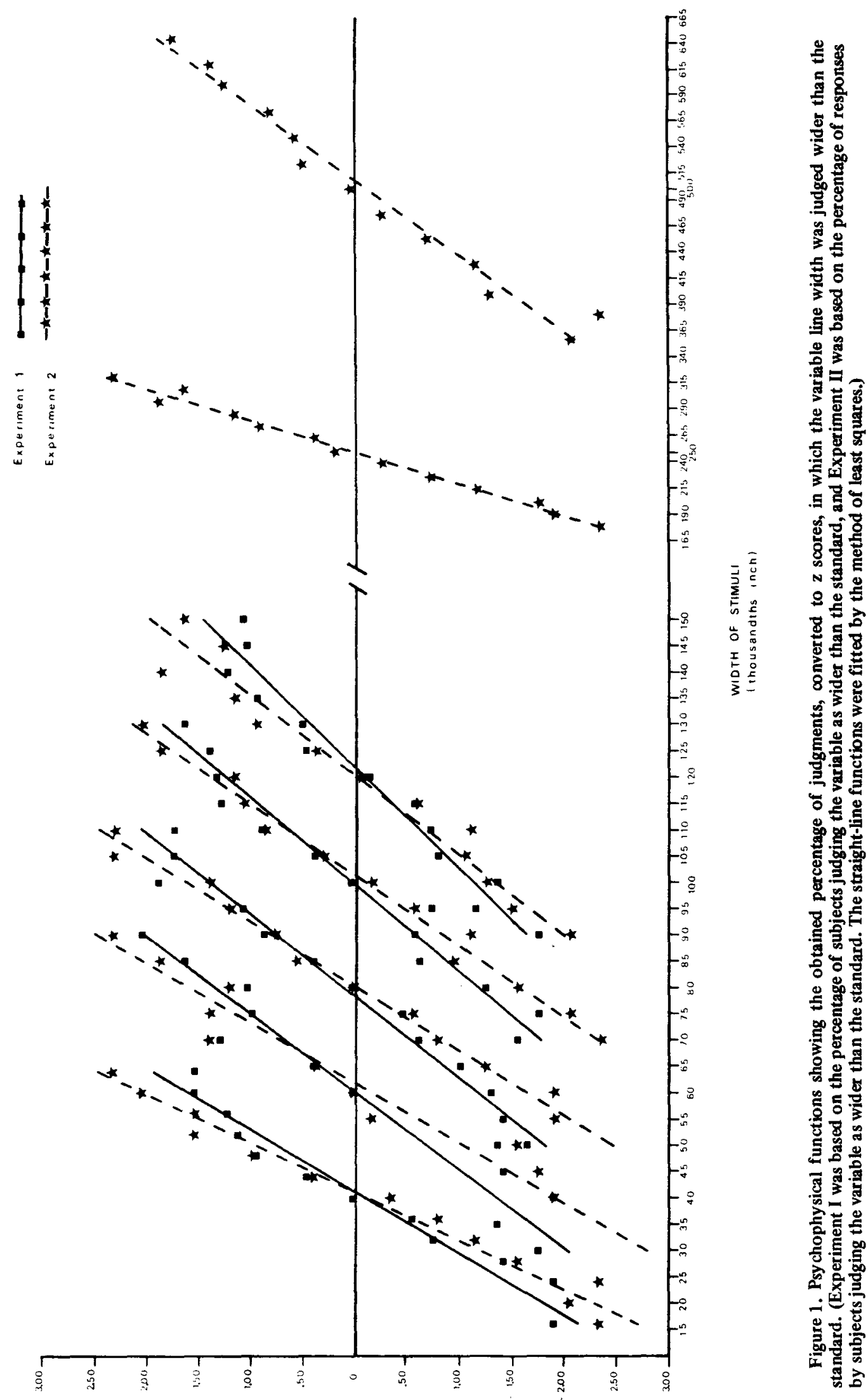
Table 2

Age, Sex, Grade Level, and Visual Acuity of Subjects in Experiment II

\begin{tabular}{cccccc}
\hline Subjects & Age $(\mathrm{yr})$ & Sex & $\begin{array}{c}\text { Functional } \\
\text { Grade } \\
\text { Level }\end{array}$ & \multicolumn{1}{c}{ Left Eye } & Risual Acuity \\
\hline $\mathrm{S}_{1}$ & 9.42 & $\mathrm{~F}$ & 2 & Light Perception & Counts Fingers $\left(1^{\prime}\right)$ \\
$\mathrm{S}_{2}$ & 13.42 & $\mathrm{~F}$ & $\mathrm{C}$ & Counts Fingers $\left(10^{\prime \prime}\right)$ & Counts Fingers $\left(10^{\prime \prime}\right)$ \\
$\mathrm{S}_{3}$ & 17.25 & $\mathrm{~F}$ & 12 & Light Perception & Prosthesis \\
$\mathrm{S}_{4}$ & 11.08 & $\mathrm{M}$ & $\mathrm{M}$ & Light Perception & Light Perception \\
$\mathrm{S}_{5}$ & 12.83 & $\mathrm{M}$ & 12 & Light Perception & $20 / 200$ \\
$\mathrm{~S}_{6}$ & 18.33 & Light Perception & Light Perception \\
\hline
\end{tabular}

previous study. and two larger standards, $.25 \mathrm{in.}(.64 \mathrm{~cm})$ and .50 in. $(1.27 \mathrm{~cm})$ were added. For the $.25-\mathrm{in} .(.64 \mathrm{~cm})$ standard, the variables were separated by $.012-i n .(.03 \mathrm{~cm})$ steps; for the .50 -in. $(1.27 \mathrm{~cm})$ standard by .024 in. $(.061 \mathrm{~cm})$ steps. The seven standard lines and their comparisons resulted in 91 pair-comparison trials.

Design and Procedure. The six subjects were selected by the administrative staff of the Kentucky School for the Blind on the basis of their availability, and each subject was paid for his participation at the rate of $\$ 1$ per session. Bonus money was paid at the end of each sixth session at the following rates: Session 6, \$2; Session 12. \$3: Session 18. \$4; Session 24, \$5, Consequently, each subject earned $\$ 38$. Initially, the six subjects were interviewed as a group and were given complete information about the rates of pay. bonus money, nature of the task, and its duration.

Each subject was given a different random order of the 91 pair comparisons during each session. Both the order of presentation and the position (left or right) of the standard and comparison were rand omized. Each subject was tested individually for three sessions per week for 8 weeks.

The same procedure was used as in Experiment I. At the beginning of each session, and periodically throughout a session, the subject was reminded to pick the thicker of the two lines. An opaque screen was placed between the experimenter and all subjects to eliminate their use of their residual vision. The subject had to slip his hand under the screen in order to make contact with the stimuli.

\section{Results}

The basic data were the percentage of trials in which the comparison stimuli were judged wider than the standard. A tabulation of the total number of correct responses was made for each subject for each standard (trials in which the standard was compared with itself were eliminated) and these data were used in an analysis of variance on the differences between sex (males vs. females). trials (1-12 vs. 13-24) and standards (seven standards). Males vs. females constituted a between-subjects variable, while trials and standards were within-subjects variables. The only significant effect was associated with trials (1-12 vs. 13-24) $(\mathrm{F}=33.60 ; \mathrm{df}=1,4 ; \mathrm{p}<.01)$. The mean percentage of correct responses for Trials 1-12 was $86.64 \%$ and for Trials 13.24 was $88.62 \%$. All six subjects showed small increases in performance over the 24 trials.

Since there were no significant differences between males vs. females, the data were combined for each standard and psychometric functions were obtained by the method of least squares. Figure 1 shows the psychometric functions for each standard, with the abscissa showing the width of each line in thousandths of an inch and the ordinate showing the obtained percentage of responses. converted to $\mathrm{z}$ scores, where the variable was judged larger than the standard. Inspection of the functions show that the PSEs for each standard were relatively unbiased. The correlations between the obtained percentages converted to $z$ scores and the line of best fit ranged from $r=.97$ to $r=.99$. showing an excellent fit to a straight-line function. Inspection of Figure 1 shows that discrimination was consistently better in Experiment II across all standards common to both experiments.

The $50 \%$ and $90 \%$ difference thresholds were obtained by use of the regression equations. Weber fractions were then computed and are shown in Table 1. Inspection of Table 1 shows that, for both the $50 \%$ and $90 \%$ thresholds, the proportion of change necessary to discriminate a line width as greater than the standard decreased as the standard became larger over the range of standards from $.04 \mathrm{in} .(.10 \mathrm{~cm})$ to $.25 \mathrm{in} .(.64 \mathrm{~cm})$. For the largest standard [.50 in. $(1.27 \mathrm{~cm})]$, the Weber fractions for both the $50 \%$ and $90 \%$ thresholds increased. suggesting a $U$-shaped function. A comparison of the Weber fractions for the standards common to both experiments shows that discrimination was consistently better with the repeated-trials procedure as compared to the "many-subjects" procedure. The ratio of the Weber fractions for the .04 in. $(.10 \mathrm{~cm})$ standard to the $.12 \mathrm{in} .(.30 \mathrm{~cm})$ standard for both the $50 \%$ and $90 \%$ thresholds were 1.78 and 1.84 , which compares favorably with the ratios obtained in Experiment I (1.82).

\section{Discussion}

Using the more traditional repeated trials procedure, the same relationship between the Weber fractions and size of standard was obtained, as in the first study; namely, as line width increased from $.04 \mathrm{in.}(.10 \mathrm{~cm})$ to $.12 \mathrm{in} .(.30 \mathrm{~cm})$, the Weber fractions decreased. Although the relative values of the Weber fractions in the second study were equivalent to the values obtained in the first study. the absolute values were lower. It is to be expected that 
the absolute values in the second experiment would be lower considering the subjects had considerably more practice over an extended period. Support for this interpretation comes from the fact that the subjects showed increasing accuracy and decreasing variability over the 24 trials. The two larger standards used in this experiment indicated that the Weber fractions approach asymptote at about .25 in. $(.64 \mathrm{~cm})$. Whether the increasing Weber fractions for both the $50 \%$ and $90 \%$ thresholds for the $.50-i n$. $(1.27 \mathrm{~cm})$ standard are indicative of a U-shape function or represent random variation around an asymptotic value cannot be determined without another study extending the size of the standards. As in the first study, there were no differences between males or females.

The major question is: What are the factors that account for the decreasing Weber fractions as line width increased up to $.12 \mathrm{in} .(.30 \mathrm{~cm})$ and for the abrupt increase in the Weber fraction for the largest standard? One factor concerns the amount of cutaneous tissue being stimulated in the finger. As the line width increased, the amount of cutaneous tissue simultaneously stimurated would increase. Consequently, as more receptor cells are stimulated, it could have provided the basis for greater accuracy of discrimination. One implication of this hypothesis is that, for a given subject, the decelerating function should asymptote at a value equal to the maximum area of cutaneous tissue that could be simultaneously stimulated. This value might be characterized as "fingerprint width."

There is no direct evidence from these experiments concerning the relationship between "fingerprint width" and accuracy of discrimination. However, it was observed during the course of the experiment that some of the larger line widths in the $.25-i n .(.64 \mathrm{~cm})$ set and almost all of the line widths in the .50-in. $(1.27 \mathrm{~cm})$ set exceeded the finger widths of the subjects. It could be inferred that, since the maximum amount of cutaneous tissue was being stimulated by the largest variables, the psychophysical function would asymptote somewhere between the $.25 \mathrm{in}$. $(.64 \mathrm{~cm})$ stand ard and the .50 -in. $(1.27 \mathrm{~cm})$ standard. However, the size of the largest standard and most of its variables exceeded the "fingerprint width" of the subjects, and the amount of tissue being stimulated would thus be a constant. Consequently, this factor could not account for the increased Weber fraction for the $.50 \mathrm{in.}(1.27 \mathrm{~cm})$ standard. Since active touch was being used, rather than passive touch, the subjects may have changed their inspection strategies, such as using slight lateral movements, and/or changed the dimension on which they were discriminating, such as extent of lateral finger movement from edge to edge across the width of the line. Both of these factors could account for the increased Weber fraction for the .50 -in. (1.27) standard.

From a practical standpoint, the results of this study can be applied to the practical problems of blind students who use tactile graphic displays and the tactile symbol legibility problem mentioned previously. First, a set of highly discriminable lines, extending from below .04 in. $(.10 \mathrm{~cm})$ to beyond .50 in. $(1.27 \mathrm{~cm})$ can be calculated from the data using the psychophysical functions and the Weber fractions. The set of lines thus obtained could be used as a standard set of lines for a variety of tactile displays (maps, graphs, diagrams). Second, using a table of the Weber fractions for each standard, one could determine how discriminable two line thicknesses were, or determine the value a line thickness would need to be in order to be discriminable from a line not used as a standard in the original study. Third, as Nolan and Morris (1971) state, "It is desirable, particularly in educational settings, that blind children have access to the same maps in their textbooks and elsewhere as do sighted children. Attainment of this goal is made difficult because of the following paradox. In comparison to that for vision, the perceptual span for touch is extremely limited making the task of reading maps far more difficult and prolonged. Consequently, tactual maps should be as small as possible. In comparison to that for vision, tactual acuity is much more coarse requiring tactual figures to be much larger than visual figures in order to be discriminated. This requires tactual maps to be much larger than visual maps if the same information is to be presented" (p. 75). Consequently, knowing the smallest differences that are highly discriminable would begin to contribute toward a solution to the tactile display size-legibility problem.

The discriminatory capacity for the width of lines represents only one of the numerous parameters that can be studied using active touch with small tactile stimuli. For example, several studies have been conducted investigating the micro- and macroscopic surface structure of tactile stimuli in the perception of roughness (Lederman. 1974; Lederman \& Taylor, 1972; Taylor \& Lederman. 1975).

For both theoretical as well as obvious practical applications of the research findings, other parameters such as distances between points, gaps between lines, difference in heights of lines, curvature, angularity, and numerosity, to mention a few, could be investigated. The practical problems for the blind student reading graphical displays suggests that an area of research which might be called active tactile nicropsychophysics is needed. 


\section{REFERENCES}

BerLA: E. P. Behavioral strategies and problems in scanning and interpreting tactual displays. New Outlook for the Blind, 1972. 66. 277.286 .

Fertsch, P. Hand dominance in reading braille. American Journal of Psychology, 1947, 60, 335-349.

KLING, J. W.. \& Riggs, L. A. Woodworth \& Schlosberg's Experimental psychology (erd ed.). New York: Holt, Rinehart and Winston. 1971.

LEDERMAN. S. J. Tactile roughness of grooved surfaces: The touching process and effects of macro- and microsurface structure. Perception \& Psychophysics. 1974. 16. 385-395.

Lederman, S. J.. \& TAYLor. M. M. Fingertip force, surface geometry, and the perception of roughness by active touch. Perception \& Psychophysics. 1972. 12.401-408.

Nolan, C. Y.. \& Kederis, C. J. Perceptual factors in braille word recognition. American Foundation for the Blind Research Series. No. 20, 1969

Nolan. C. Y.. \& Morris, J. E. Improvement of tactual symbols for blind children. Final report. Project No. 5-0421.
Grant OEG-32-27.0000-1012. American Printing House for the Blind. Louisville. Kentuck:, 1971

Morris, J. E.. \& Nolan. C. Y. Minimum sizes for areal type tactual symbols. Intermational Joumal for the Education of the Blind. 1963. 11. 50-54.

TAYlor, M. M.. \& Lederman. S. J. Tactile perception of grooved surfaces: A model and the effect of friction. Perception \& Psychophysics. 1975. 17, 23-36.

ZIGLER, M. J.. \& NoRTHRUP, K. M. The tactual perception of form. American Jourmal of Psychologi. 1926. 37. 391-397.

\section{NOTE}

1. The $90 \%$ threshold has been adopted as a minimum standard criterion for discriminability of tactile materials by the American Printing House for the Blind, which produces numerous educational aids for visually handicapped children.

(Revision accepted for publication April 25. 1975.) 